\title{
DISCUSSION
}

\section{Exploiting opportunities computers provide to deliver content people can use in context}

\section{A response to 'Accessibility and adaptability of learning objects: responding to metadata, learning patterns and profiles of needs and preferences' 1}

\author{
Andy Heath* \\ Sheffield Hallam University, UK
}

As a contributor to the IMS (2004) AccessForAll work and co-editor of its development in ISO (2005a, b, c), I find it pleasing to see our work being implemented and enlightening to see some of the ways that ideas one has worked on are interpreted, and discover these are not always ways that were intended. Although I am fortunate enough to have been able to participate in the AccessForAll work, the views I present here are my own and may or may not reflect those of my co-editors in that work.

I shall address some of the fundamental ideas in the paper in the light of the AccessForAll approach.

The AccessForAll work contains two central ideas:

1. That resources are matched at delivery time with functional requirements of learners or users that are relevant to that immediate context.

2. That the components that comprise a delivered resource can be assembled from distributed sources.

These ideas work in concert to deliver an experience that can be accessed by the individual in that context. Neither idea on its own is sufficient, and applying each without serious care for the goals in their design can have interesting, possibly undesirable, consequences. (There is in fact a third idea-that distributed resources and adaptations have associated metadata that enable us to find them, but space precludes addressing that here.)

The first of these ideas, matching resources to context, is not completely new. It is arguable that since all computer systems aim to be usable they all set out to achieve

*Axelrod Research and Consultancy, 51 Paterson Close, Stocksbridge, Sheffield, UK. Email: AndyHeath@axelrod.plus.com 
this but the mechanism available is informal. Without a standard way to record preferences and requirements, each application or system will take its own best guess at such a data set and the result will be that they each will have a different data set in mind. The mere existence of a standard format, such as the AccessForAll Personal Needs and Preferences profile, can provide a target for applications to aim for. Thus one might argue, as I interpret that the Learning Patterns paper does, that an author providing content suitable for some environment can identify a generic set of preferences that apply to that environment (such as an 'audio profile'). That process and set of preferences can then guide the author's thinking.

While this is progress, a number of things are missing from the argument:

1. The existence of a generic anonymous set of preferences is not the same as the preferences of an individual learner. It is like a 'type' statement for a variable as can occur in a computer program. It is not an example value. At some level it will hide the individual requirements of a user. Using it is like a designer of roads intended for cars reading the blueprints for a bicycle and then designing cycling facilities: at some level he/she will get it wrong, unless he/she asks the cyclists who will use it to participate in the design.

2. It is not distinct from the notion of a Universally Accessible Resource, which the paper rightly points out is a flawed notion because it is not achievable. The world already has many design patterns for accessible design. There are, for example, numerous sets of guidelines, the most notable being those of the Web Accessibility Initiative Web Content Accessibility Guidelines (WAI, 2005). On their own, they do not guarantee an accessible result (Kelly et al., 2005); indeed, used alone they present the danger that a designer will claim that they do and believe they have 'done' accessibility. Design patterns and guidelines are useful, but more work is needed to explore how they may be used in practice without being misused.

This brings us to the second of the ideas in AccessForAll and the reasons it is essential.

On the one hand, we argue that accessibility for disabled persons is a field where expertise is specialised, difficult to learn and rare. This is the case because of the comparatively small numbers of persons obviously disabled by current environments and the great diversity of assistive technology. On the other hand, we argue that we are doing 'Access' for everyone, and it needs to be generic to profit from benefits of scale. Both of these pictures are correct in some sense, but the first is where we are currently and the second is where we are aiming to get to. Technology always changes, and business drivers and the wish for technology to serve the needs of people in a better way ensure we stay always at the leading edge of change. Consequently, it is probable that these two pictures will remain the case and our path will always be to try and go from the first to the second. In striving to do this, there is the danger that the user's needs will be forgotten or overridden by those of the vendor, system or content supplier or organisation. Using design templates carries the danger that they can be used to meet only the needs of supplier organisations and avoid meeting the real needs of individuals. 
The second AccessForAll concept is based around the view that whatever we do we will get it wrong and need to put it right, but that this is OK. We hold the tenet that it is unreasonable to expect all authors to have specialised accessibility expertise. Whatever tools content authors use or systems provide, they will have faults. Just as a Universally Accessible Resource is not something that can exist, neither can there be an authoring tool that creates one. So for any interesting learning object, however good it is at being accessible to all, it is probable there will be some persons for whom it is not accessible. Making it accessible for such a person will require adaptations. The job of creating adaptations is often best done by someone with expert knowledge of that user's accessibility requirements and of the context. Thus the person who does that may be outside the organisation that originally produced the resource. In a university, it may be a disability service. In the world of Hollywood films, it may be a specialised captioning service. In an AccessForAll world, where components of a learning object can be assembled at or close to run time and may come from inside or outside the organisation where the resource was authored, the person that authored the adaptation may not be the person that had the design template in mind. It seems that the notion of design templates alone may require that all authors become Accessibility-Aware. This is not likely to happen.

I must ask, then, what the place of Design Templates is and whether they are of any value.

It seems clear, as the authors describe, that they can be useful in practice. Teachers do use design templates-it seems they know what patterns will and will not work in the classroom and use those patterns. But they know also when to throw the template away and respond to an individual in a different way. It seems to me that technology is not yet very good at providing ways to do that. Sometimes we need to just break all the rules and do something different because it works.

A full treatment of AccessForAll would discuss issues around metadata authoring and searching, blended learning, granularity, aggregation formats and mapping between them, the semantic web and other complex areas of work in development in various places. While there is not space for that here, it is interesting to think briefly about online resources, non-online resources and matching authored resources to context. It seems to me that books are an example of the use of Design Patterns that has been well explored-and they work. An author can use a standard pattern to write a book without knowing the intimate details of the context in which the book will be read. The paper's treatment of design patterns seems very similar. But if we stick with this model we may be bending only to the wishes of those who want to sell us content and tools and not getting the best use out of computers. Perhaps by coping with the complexities of really matching not to general templates but to individual requirements, we can get more out of computers than this. In this analysis it seems to me that design templates in this context represent a half-way house between the world where we say to learners 'here is what I think you need to access this', and a world where learners say 'here is what I need to access this'. As such they may be a good step, but more work is needed to determine how they can be used to best benefit everyone- - that is, to use them in context and with distributed adaptations. 
I will end this response by posing some questions that might help research towards the goal of using Design Templates in broad contexts that can meet individual requirements:

1. What is the value of talking about groups of learners?

2. How can we ensure Design Templates are not misused?

3. How can Design Templates cater for repressed demand-where a user may only select from what is available and not know to say 'I want this'?

4. How can Design Templates be used across systems and organisations in ways that remain responsive to real individual requirements?

\section{Note}

1. Published in Alt-f (2006), 14(1), 117-129.

\section{References}

IMS (2004) IMS AccessForAll Metadata specification. Available online at: http://www.imsglobal.org/ accessibility/ (accessed 22 February 2006).

ISO (2005a) Individualised adaptability and accessibility in e-learning, education and training. Part 1: framework, ISO IEC JTC1 SC36 Available online at: http://jtc1sc36.org/doc/36N1139.pdf (accessed 22 February 2006).

ISO (2005b) Individualised adaptability and accessibility in e-learning, education and training. Part 2: Access For All Personal Needs and Preferences Statement. Available online at: http://jtc1sc36.org/ doc/36N1 140.pdf (accessed 22 February 2006).

ISO (2005c) Individualised adaptability and accessibility in e-learning, education and training. Part 3: Access For All Digital Resource Description. Available online at: http://jtc1sc36.org/doc/ 36N1141.pdf (accessed 22 February 2006).

Kelly, B., Phipps, L. \& Howell, C. (2005) Implementing a holistic approach to e-learning accessibility, in: J. Cook \& D. Whitelock (Eds), Exploring the frontiers of e-learning: borders, outposts and migration, ALT-C 2005 12th International Conference Research Proceedings, ALT Oxford.

WAI (2005) Web Accessibility Initiative Web Content Accessibility Guidelines 2.0 Working Draft. Available online at: http://www.w3.org/WAI/ (accessed 22 February 2006). 